\title{
Indicadores psicosociales de motivación del consumo de marihuana y/o cocaína
}

\author{
Raúl García-Aurrecoechea*; Rogelio Díaz-Guerrero**; Isabel Reyes-Lagunes***; \\ María Elena Medina-Mora***; Patricia Andrade-Palos***; Lucy María Reidl Martínez*** \\ * Departamento de Investigación Clínica y Epidemiológica, Centros de Integración Juvenil, A.C. \\ ** (1918-2004) Facultad de Psicología, Universidad Nacional Autónoma de México \\ *** Facultad de Psicología, Universidad Nacional Autónoma de México \\ *** División de Investigaciones Epidemiológicas y Sociales, Instituto Nacional de Psiquiatría "Ramón de la Fuente". \\ Enviar correspondencia a: \\ Raúl Aurrecoechea. Correo electrónico: raurre@hotmail.com
}

\section{RESUMEN}

Con el fin de identificar indicadores de protección y riesgo del consumo de marihuana y/o cocaína en el contexto de la teoría de la motivación de Maslow, se compararon los significados psicológicos de cinco conceptos clave (familia, amigos, yo, cocaína y marihuana) en jóvenes del sexo masculino, usuarios de marihuana, cocaína y ambas drogas que solicitaron tratamiento y estudiantes no consumidores de drogas. Los resultados mostraron diferencias en la evaluación en los cinco conceptos entre los grupos, encontrándose entre los no usuarios mejores evaluaciones en la familia, los amigos y el yo (en acuerdo con postulado de psicopatogénesis de la teoría psicosocial de la motivación de Maslow), así como peores evaluaciones de las drogas. Como indicadores de protección de la familia, los amigos y el yo, se encuentra la satisfacción de necesidades deficitarias e indicadores que sugieren la activación del circuito de gratificación, mientras que como indicadores de riesgo, sobresalieron componentes negativos incompatibles con los indicadores de protección. Entre los indicadores de riesgo asociados con las sustancias, se identificaron componentes positivos que muestran la activación del circuito de recompensa. Los resultados son congruentes con los factores reportados por la literatura, considerándose importante explorarlos más a fondo, con el fin de identificar necesidades de prevención y tratamiento para cada grupo de usuarios

Palabras clave: marihuana, cocaína, motivación, Maslow, psicosocial, indicadores.

\section{ABSTRACT}

In order to identify marihuana and/or cocaine use protective and risk indicators within the context of Maslow's theory of motivation, we compared the psychological meanings of five key concepts (family, friends, self, cocaine and marihuana) among young male users of marihuana and cocaine, and users of both drugs who requested treatment, and non-drug user students. Results showed differences in the evaluation of five key concepts in the groups, where we found higher evaluations of family, friends and self in non-users (concordant with the psycho-pathogenesis postulate of psychosocial Maslow motivational theory), in addition to lower evaluations of drugs. Taking family, friends, and self as protective indicators, we find satisfaction of deficit needs and indicators that suggest encephalic reward circuit activation, whereas risk indicators are mostly negative components, incompatible with protective indicators. Among risk indicators associated with the substances, we identified positive components that show reward circuit activation. Results correspond with the factors reported in the literature, and we consider it important to explore them in greater depth in order to identify the prevention and treatment needs of each drug user group.

Key words: marihuana, cocaine, motivation, Maslow, psychosocial, indicators.
$E$ ntre los efectos farmacológicos más importantes en el nivel neuronal debidos al consumo de drogas (como la cocaína y la marihuana) se encuentra la activación del circuito de recompensa encefálico, relacionada con una amplia gama de sensaciones de placer, disfrute y satisfacción (Guisa, Díaz-Barriga, Sánchez-Huesca, y Sousa, 2001; Ramos y Fernández,
2000; Volkow, Fowler y Wang, 2003). Esta información ha sido útil para el conocimiento de las adicciones y ha ayudado a orientar investigaciones hacia la identificación de las sensaciones de satisfacción particulares, que son buscadas y experimentadas por los consumidores y hacia las relaciones que sostienen con otras variables. 
Entre éstas, se encuentran los factores asociados, los cuales se pueden dividir en macrosociales, microsociales e individuales (Hawkins, Catalano y Miller, 1992), siendo los dos últimos el campo de intervención de la psicología. Estos factores pueden cubrir un papel de de protección (cuando tienden a evitar que ocurra el consumo o las recaídas) y de riesgo (cuando favorecen la aparición del uso de drogas).

Entre los factores microsociales tienen un peso importante aquellos relacionados con la familia, se ha identificado que una familia nutriente, que también pone límites, tiene un efecto de protección, mientras que se ha asociado con riesgo a las normas inconsistentes (Hawkins, Catalano y Miller, 1992; Munist, Santos, Kotliarenco, Suárez, Infante y Grotberg, 1998; Pérez y Mejía, 1998) la violencia y el conflicto intrafamiliar (Rodríguez-Kuri, 2004). También se ha encontrado que un factor de riesgo importante es el consumo de drogas en la familia (Hawkins et al., 1992; MedinaMora, Cravioto, Villatoro, Fleiz, Galván-Castillo y TapiaConyer., 2003; Munist et al., 1998; Rodríguez-Kuri, 2004.

Otro grupo de factores microsociales asociados de consumo de drogas lo constituyen los amigos, habiéndose asociado con riesgo tanto la presencia de conductas antisociales como el consumo de drogas o su permisividad (Medina-Mora et al, 2003; Munist et al, 1998; Rodríguez-Kuri, Arellanes, Díaz-Negrete y González-Sánchez, 1999).

Por parte de los factores de tipo individual sobresalen como protección una buena autoimagen y autovaIoración (Rodríguez-Kuri y Pérez-Islas, 2002), mientras que como riesgo, aparece la depresión (González-Sánchez, García-Aurrecoechea y Córdova, 2004; MedinaMora et al, 2003), así como los trastornos de conducta (Rodríguez-Kuri et al, 1999).

Por otra parte, la búsqueda de sensaciones se ha ubicado como factor de riesgo del uso de marihuana, en particular los viajes (Gracia, Saldívar y Contreras, 2002).

Un estudio reciente, realizado con las mismas cuatro muestras de este estudio, parece confirmar el postulado de la psicopatogénesis de la teoría de la motivación de Maslow (1954/1970a y b). Este estudio exploró modelos predictivos del uso de marihuana, cocaína o ambas drogas y se basó en necesidades deficitarias identificadas como relevantes para la cultura mexicana (Díaz-Guerrero y Díaz-Loving, 2001). El estudio detectó que la satisfacción de determinadas necesidades conformaba elementos de protección del consumo (GarcíaAurrecoechea y Gracia, 2004)

De tal manera, la satisfacción de las necesidades de salud y de cariño se conformaron como protectoras del policonsumo (marihuana y cocaína); la satisfacción de las necesidades de tranquilidad y éxito en la familia como protectoras del uso de cocaína y; la satisfacción de las necesidades de éxito y respeto como protectoras del uso de marihuana (García Aurrecoechea y Gracia, 2004).

En otro orden de ideas, con respecto a los significados psicológicos de grupos sociales a partir de asociaciones libremente expresadas por grupos de individuos se encuentra basado en Szalay y Bryson (1974), quienes diseñan una técnica llamada Análisis Asociativo de Grupo (AAG), la cual ha probado ser capaz de medir los significados psicológicos con una eficiencia comparable a aquella de otros métodos, entre los que se encuentran la similitud de rangos y la adaptación de palabras del diferencial semántico.

El AAG Ileva a cabo el análisis de cientos de asociaciones libremente expresadas de manera espontánea ante los temas estímulo, mediante muestras de tamaño mediano, en las que cada sujeto aporta entre cinco y diez asociaciones (Díaz-Guerrero y Szalay, 1993), organizando la información producida por medio de Pesos Relativos (PR) aplicados a las respuestas (desde seis puntos a la primera, hasta uno a la décima), a partir de los cuales se obtienen las Puntuaciones de Predominio (PP), al sumar los PR de las palabras repetidas, de tal manera que las PP determinan las asociaciones o temáticas con mayor número de acuerdos.

Otra medida utilizada por el AAG es el Indice de Dominancia Evaluativa (IDE), el cual evalúa la relación de respuestas con connotaciones positivas o negativas en una escala de -100 a 100. Se obtiene después de solicitar a un grupo de especialistas en el tema que funjan como jueces, para clasificar las asociaciones en Componentes Subjetivos (CS) Positivos, Negativos y Neutros. La formula para obtener el IDE es:

$$
I D E=\frac{\text { PR Positivos }- \text { PR Negativos } \times 100}{\text { Total de PR }}
$$

Con base en lo anteriormente expresado, los objetivos del estudio fueron comparar los significados psicológicos de las palabras familia, amigos, yo, marihuana y cocaína en tres grupos de usuarios actuales (de marihuana, cocaína y ambas sustancias) y un grupo de no consumidores de drogas y discutir los resultados con base a los factores de riesgo y protección reportados a partir del postulado de psicopatogénsis de la teoría de la motivación de Maslow (1954/1970b).

\section{MÉTODO}

Se llevó a cabo un estudio transversal, ex-post-facto con cuatro muestras independientes: 1) usuarios de marihuana, 2) de cocaína, o 3) de ambas drogas y 4) no usuarios de drogas. Las muestras de consumidores se ubicaron entre solicitantes de tratamiento a Centros de 
Integración Juvenil, A.C., durante el primer trimestre de 2003, verificando que no presentaran uso de solventes inhalables, heroína, o metanfetamina, mientras que los no usuarios de drogas fueron captados entre estudiantes de secundaria, preparatoria y profesional en último trimestre de 2002, los cuales reportaron no consumir drogas de curso ilegal y, en caso de haberlas consumido, que hubiera sido de forma experimental (cinco veces o menos).

Se obtuvo información de 295 adolescentes y adultos jóvenes del sexo masculino de la Ciudad de México, en un rango de edad entre 13 y 29 años, de los cuales 151 fueron no usuarios de drogas de curso ilegal, 47 usuarios actuales (uso en el último mes) de marihuana, sin uso en el último año de cocaína ni otra sustancia de curso ilegal, 47 usuarios actuales de cocaína, sin uso en el último año de marihuana ni otra sustancia de curso ilegal y 50 usuarios actuales de marihuana y cocaína, sin uso en el último año de ninguna otra sustancia de curso ilegal. Se puede apreciar un sobre-muestreo del grupo de no usuarios, dado que fue más sencillo el acceso a ellos, lo cual prestó a la oportunidad detectar un mayor espectro de indicadores de protección:

El instrumento consistió de identificación sociodemográfica, patrón de consumo de sustancias y significados psicológicos asociados con: 1) la familia, 2) los amigos, 3) el yo, 4) la marihuana y 5) la cocaína. Los Pesos Relativos, las Puntuaciones de Predominio y los Indices de Dominancia Evaluativa (IDE) fueron obtenidos de acuerdo con Díaz-Guerrero y Szalay (1993). La obtención de los IDE se realizó con el apoyo de un grupo de jueces, compuesto por cinco psicólogos especializados en adicciones, que clasificaron las asociaciones de cada palabra estímulo en componentes subjetivos positivos, negativos o neutros. En el texto se analizan las Puntuaciones de Predominio de los Componentes Subjetivos positivos o negativos relacionados con el riesgo o protección del consumo de drogas. En el caso de las sustancias, se destacan los componentes subjetivos positivos de riesgo del consumo presentes de los consumidores de las sustancias.

En los análisis estadísticos se utilizó el programa estadístico SPSS.10, con el cual se aplicaron cinco pruebas de ANOVA de una sola vía (con post hoc de Tukey).

\section{RESULTADOS}

Para la palabra estímulo familia, los no consumidores reportaron 410 asociaciones, los poliusuarios de marihuana y cocaína 236, los de cocaína 136 y los de marihuana 206. Sobre los amigos, los no consumidores produjeron 391, los poliusuarios 257, los usuarios de cocaína 176 y los de marihuana 213. Acerca de la palabra yo, los no consumidores de drogas aportaron 470 asociaciones, los poliusuarios 242 , los usuarios de cocaína 243 y los de marihuana 248.

Sobre la cocaína, los no consumidores de drogas reportaron 395 asociaciones, los poliusuarios 220, los usuarios de cocaína 202 y los de marihuana 132. Para la marihuana los no consumidores produjeron 358 asociaciones, los poliusuarios 224; los usuarios de cocaína, 121 y los de marihuana 200.

Familia. Todos los grupos encuentran los conceptos de unión, apoyo, amor y comprensión asociados con la familia, sin embargo, a diferencia de los otros grupos, los no consumidores de drogas también la relacionan con amistad, ser feliz y la consideran alegre y honesta (Tabla 1), además que también incluyen el regaño, siendo este último ubicado por los jueces como componente subjetivo negativo.

Entre las carencias al interior de los grupos de consumidores, se observa que adolecen en distintas medidas de determinados componentes subjetivos positivos como: respeto sinceridad, convivencia, educación, responsabilidad, trabajo y de asociarla con buenas personas (Tabla 1).

Tabla 1. Indicadores de protección y riesgo del consumo de marihuana, cocaína o ambas drogas relacionados con la FAMILIA

\begin{tabular}{|c|c|c|c|}
\hline \multicolumn{4}{|c|}{ Palabra estímulo FAMILIA } \\
\hline \multicolumn{2}{|c|}{ Componentes Subjetivos Protectores } & \multicolumn{2}{|c|}{ Componentes SubJetivos de Riesgo } \\
\hline \multicolumn{2}{|c|}{ Positivos } & & \\
\hline $\begin{array}{c}\text { feliz } \\
\text { amistosa } \\
\text { alegre } \\
\text { honesta }\end{array}$ & $\begin{array}{l}\text { respeto }{ }^{* * *} \\
\text { sinceridad } *^{* * *} \\
\text { convivencia }{ }^{* * * *} \\
\text { educada } *^{*} * * \\
\text { trabajadora }{ }^{* *} \\
\text { responsable } \\
\text { buenas personas }\end{array}$ & & \\
\hline & Negativos & & Negativos \\
\hline & regaña & $\begin{array}{l}\text { hipócrita *** } \\
\text { agresiva ** } \\
\text { mala ** }\end{array}$ & $\begin{array}{l}\text { falta de atención }{ }^{* * *} \\
\text { discusiones } \\
\text { desintegración } \\
\text { miedo * }\end{array}$ \\
\hline
\end{tabular}

* Protección o riesgo del consumo de marihuana; ** del consumo de cocaína; *** del consumo de ambas. 
Entre los componentes subjetivos relacionados con el riesgo, se destacaron los ubicados por los jueces como negativos. En particular, en los usuarios de cocaína, se destaca una asociación con la agresión, en los poliusuarios aparecen las discusiones y la falta de atención y en los de marihuana la desintegración y el miedo, mientras que en los grupos de monousuarios, se asocia con la hipocresía. Además, es notable que en ninguno de los grupos de usuarios se presentaran asociaciones relacionadas con las drogas (Tabla 1).

Amigos. Todos los grupos concordaron en asociar los amigos con la propia amistad, diversión, juego, ayuda, apoyo, sinceridad y alegría, sin embargo, los no consumidores de drogas, además los asociaron con unión, respeto, cariño y amabilidad. Al interior de los grupos de consumidores se encuentra la ausen- cia de ciertas asociaciones, tales como comprensión, convivencia, confianza y estudio; tampoco pensaron que fueran buenos, ni "chidos" (Tabla 2).

Entre los componentes positivos de riesgo, para tres grupos de consumidores, se encontraron pláticas, "cotorreo" y fiesta; para los usuarios de cocaína y los poliusuarios trabajo y compañía; para los de cocaína y para los de marihuana la hermandad. Otros componentes de riesgo particulares es que sean muchos, tranquilidad, influencia, bailar, creer que son para siempre, quererlos (los quiero), compartir y entre usuarios de marihuana salidas, paseos o viajes.

Destaca también, para todos los grupos de usuarios, el "relajo" como componente subjetivo neutro de riesgo (Tabla 2).

Tabla 2. Indicadores de protección y riesgo del consumo de marihuana, cocaína o ambas drogas relacionados con los AMIGos

\begin{tabular}{|c|c|c|c|}
\hline \multicolumn{4}{|c|}{ Palabra estímulo AMIGOS } \\
\hline \multicolumn{2}{|c|}{ Componentes SubJetivos Protectores } & \multicolumn{2}{|c|}{ Componentes SUBJETIVOS DE RiesGo } \\
\hline & Positivos & & Positivos \\
\hline \multirow[t]{5}{*}{$\begin{array}{l}\text { unión } \\
\text { respeto } \\
\text { cariño } \\
\text { amabilidad } \\
\text { "chidos" }\end{array}$} & $\begin{array}{l}\text { comprensión } * * \\
\text { convivencia }{ }^{* *} \\
\text { son buenos } * * * * \\
\text { confianza } * * * \\
\text { estudio } * * *\end{array}$ & $\begin{array}{l}\text { pláticas } \\
\text { cotorreo } \\
\text { fiestas }\end{array}$ & $\begin{array}{l}\text { bailar ** } \\
\text { hermandad *** } \\
\text { son para siempre ** } \\
\text { influencia ** } \\
\text { los quiero * } \\
\text { compartir * } \\
\text { salidas, paseos, viajes * } \\
\text { trabajo *** } \\
\text { compañía *** } \\
\text { son muchos *** } \\
\text { tranquilidad *** }\end{array}$ \\
\hline & & \multicolumn{2}{|r|}{ NeUtro } \\
\hline & & \multicolumn{2}{|r|}{ "relajo" } \\
\hline & & \multicolumn{2}{|r|}{ Negativos } \\
\hline & & $\begin{array}{l}\text { usan drogas } \\
\text { son drogadictos } \\
\text { no existen }\end{array}$ & $\begin{array}{l}\text { hipócritas } * * * * * \\
\text { convenencieros } * * * * * \\
\text { deshonestos ** } \\
\text { traición }{ }^{*}\end{array}$ \\
\hline
\end{tabular}

* Protección o riesgo del consumo de marihuana; ** del consumo de cocaína; ** del consumo de ambas.

Entre los indicadores negativos de riesgo para los tres grupos de usuarios se encontraron asociaciones vinculadas con las drogas y la negación de la existencia de la palabra estímulo (no hay, no existen). Existe además hipocresía, considerarlos convenencieros, deshonestos y se presenta la traición (Tabla 2).

Autoconcepto. Se descubre que en buena medida todos los grupos se consideran buenos, les importa estudiar, la amistad, trabajar y la alegría. Pero, con diferencia de los tres grupos de usuarios, los no consumidores asociaron a su persona con respeto, libertad y cantar (Tabla 3).

Con respecto a carencias particulares en el mundo subjetivo de ciertos grupos de usuarios, se encuentra la responsabilidad, la amabilidad y el amor, así como la honestidad, la tranquilidad y la inteligencia (Tabla 3).

De manera particular, llama la atención que entre los poliusuarios no se presenta el juego ni la sinceridad y entre los consumidores de cocaína no aparece de manera importante la disposición de ayudar y se consideran fuertes (Tabla 3).

Es importante destacar que entre los componentes negativos de los usuarios con respecto a su persona se encuentra el consumo de drogas, tanto por su uso como definiéndose como usuarios: uso drogas, soy adicto, me drogo, drogadicto, mientras que los no usuarios solo hicieron referencia a tomar y fumar (al igual que los usuarios). 
Tabla 3. Indicadores de protección y riesgo del consumo de marihuana, cocaína o ambas drogas relacionados con el autoconcepto

\begin{tabular}{|c|c|c|c|}
\hline \multicolumn{4}{|c|}{ PALABRA ESTímulo YO } \\
\hline \multicolumn{2}{|c|}{ COMPONENTES SubJetivos Protectores } & \multicolumn{2}{|c|}{ Componentes SubJetivos de Riesgo } \\
\hline \multicolumn{2}{|c|}{$\begin{array}{c}\text { Positivos } \\
\end{array}$} & \multicolumn{2}{|r|}{ Positivo } \\
\hline \multicolumn{2}{|c|}{$\begin{array}{c}\text { respeto } \\
\text { libertad } \\
\text { cantar }\end{array}$} & \multirow{2}{*}{\multicolumn{2}{|c|}{ fuerte ${ }^{*} *$}} \\
\hline \multirow[t]{4}{*}{ 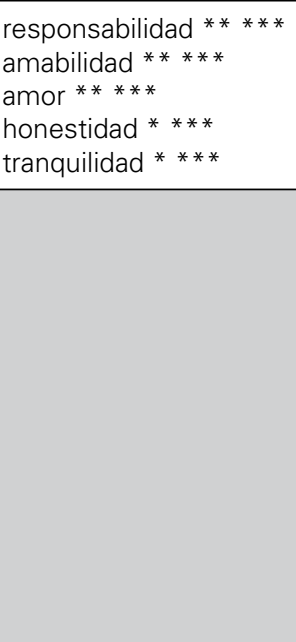 } & $\begin{array}{l}\text { inteligencia **** } \\
\text { bailar * } \\
\text { juego *** } \\
\text { sinceridad *** } \\
\text { ayudar ** }\end{array}$ & & \\
\hline & & \multicolumn{2}{|r|}{ Negativos } \\
\hline & & \multicolumn{2}{|r|}{$\begin{array}{l}\text { soy adicto } \\
\text { uso drogas }\end{array}$} \\
\hline & & $\begin{array}{l}\text { alejado }{ }^{* *} \\
\text { soledad } * * * \\
\text { triste } \\
\text { inseguro } \\
\text { tengo miedo } \\
\text { agresivo } \\
\text { insatisfecho } \\
\text { confusión } \\
\text { rebelde }\end{array}$ & $\begin{array}{l}\text { antisocial } * * * \\
\text { problemas } * * * \\
\text { depresión } * * * \\
\text { no tengo valor } * * \\
\text { no merezco *** } \\
\text { soy: malo, tarado, idiota, ignorante, un problema, cochino, } \\
\text { "rata" *** } \\
\text { estoy: mal, loco, } \\
\text { tarado *** }\end{array}$ \\
\hline
\end{tabular}

* Protección o riesgo del consumo de marihuana; ** del consumo de cocaína; ** del consumo de ambas.

Otros indicadores negativos de riesgo fueron la soledad o sentirse alejado, confusión, tristeza, y entre los poliusuarios la depresión (Tabla 3), destacándose en este grupo en particular la presencia de un bloque de conceptos auto-denigratorios, tales como: soy malo, tonto, idiota, un problema, ignorante, cochino, estoy mal, loco, tarado, no tengo valor y no merezco.

Indicadores negativos de riesgo del uso de cocaína es el miedo, en los de marihuana inseguridad, en los poliusuarios considerarse antisocial (en el sentido de no gustarle la sociedad), mientras que en los no usuarios (como en los demás grupos) sólo se presen- ta ser tímido. Además, destaca que en los usuarios de cocaína se presenta como indicador de riesgo ser agresivo y en los de marihuana rebelde (Tabla 3).

Cocaína. Todos los grupos consideraron que esta sustancia tiene que ver con la muerte, la destrucción, que es dañina y un vicio. Sin embargo, como indicadores negativos de protección de su consumo aparece considerarla una enfermedad, perjudicial, peligrosa y que no les guste. Otros indicadores negativos de protección fueron considerarla droga, mala y cara, así como, relacionarla con la soledad (Tabla 4).

Tabla 4. Indicadores de protección y riesgo del consumo de cocaína relacionados con sus significados psicológicos*

\begin{tabular}{|c|c|c|c|}
\hline \multicolumn{4}{|c|}{$\begin{array}{l}\text { Palabra estímulo COCAÍNA } \\
\end{array}$} \\
\hline \multicolumn{2}{|c|}{$\begin{array}{l}\text { Componentes SubJetivos Protectores del Consumo de } \\
\text { cocaína }\end{array}$} & \multicolumn{2}{|c|}{ Componentes SubJetivos de Riesgo del Consumo de cocaína } \\
\hline & & \multicolumn{2}{|c|}{ Positivos } \\
\hline & & \multicolumn{2}{|c|}{$\begin{array}{c}\text { me gusta, es rica } \\
\text { da placer } \\
\text { da tranquilidad } \\
\text { estar con amigos }\end{array}$} \\
\hline \multicolumn{2}{|c|}{ Negativos } & \multicolumn{2}{|c|}{ Negativos } \\
\hline $\begin{array}{l}\text { enfermedad } \\
\text { perjudicial } \\
\text { peligrosa } \\
\text { no me gusta }\end{array}$ & $\begin{array}{c}\text { droga } \\
\text { mala } \\
\text { cara } \\
\text { produce adicción } \\
\text { te lleva a la soledad }\end{array}$ & $\begin{array}{l}\text { ansiedad } \\
\text { tristeza } \\
\text { depresión } \\
\text { miedo } \\
\text { nervios } \\
\text { desesperación }\end{array}$ & $\begin{array}{c}\text { pánico } \\
\text { paranoia } \\
\text { perdición } \\
\text { se puede ir a la cárcel } \\
\text { gastar dinero } \\
\text { sin dinero }\end{array}$ \\
\hline
\end{tabular}

* Se analizan solo usuarios de cocaína, de ambas drogas (marihuana y cocaína) y no usuarios. 
Entre los indicadores negativos de riesgo del consumo de cocaína aparecieron sentimientos de ansiedad, tristeza y desesperación, apareciendo también estados alterados como la paranoia o el pánico, estar implicado en robar, o en ir a la cárcel, vincularla con lo negativo, la perdición, la depresión y con sentimientos de miedo y de "nervios" (Tabla 4).

Por su parte, entre los indicadores positivos de riesgo del consumo de cocaína se encuentra su gusto por la ésta, considerarla rica (relacionado con su sabor) y que les produzca placer, tranquilidad y ayude a estar con amigos (tabla 4).
Marihuana. Se destaca que todos los grupos consideran a la marihuana una droga apestosa y como indicadores negativos de protección de su consumo aparece relacionarla con un mal hábito, muerte, vicio, enfermedad, desesperación y nociva. Entre indicadores negativos de riesgo se encontró la presencia de problemas, sobresaliendo los de tipo familiar (tabla 5).

Entre los indicadores positivos de riesgo del consumo de marihuana, se encuentra considerar que es natural, barata, que produce tranquilidad, relajación, que es medicinal, ayuda a la diversión y a "cotorrear", produce sensaciones placer, risa, alegría y es agradable (Tabla 5).

\section{Tabla 5. Indicadores de protección y riesgo del consumo de mariHuANa relacionados con sus significados psicológicos*}

\begin{tabular}{|c|c|}
\hline \multicolumn{2}{|c|}{ PALABRA ESTímulo MARIHUANA } \\
\hline $\begin{array}{l}\text { Componentes SubJeTIVOS PROTECTORES DEL CONSUMO } \\
\text { DE MARIHUANA }\end{array}$ & Componentes SubJetivos de Riesgo del CONSUMO dE MARIHUANA \\
\hline & Positivos \\
\hline & $\begin{array}{c}\text { ayuda a la diversión } \\
\text { produce sensaciones } \\
\text { placer } \\
\text { es agradable }\end{array}$ \\
\hline Negativos & Negativos \\
\hline $\begin{array}{l}\text { nociva } \\
\text { mal hábito } \\
\text { vicio }\end{array}$ & $\begin{array}{c}\text { conflictos } \\
\text { problemas (familiares) }\end{array}$ \\
\hline
\end{tabular}

* Se analizan solo usuarios de marihuana, de ambas drogas (marihuana y cocaína) y no usuarios.

\section{Confiabilidad de los IDE de la familia, amigos y el yo}

Se obtuvo el Alfa de Cronbach de los Indices de Dominancia Evaluativa de la familia, los amigos y el yo en conjunto, resultando éste aceptable (.6553).

\section{Análisis de Varianza de los Significados Psicológicos}

La comparación de los significados psicológicos se llevó a cabo por medio de Indices de Dominancia Evaluativa (IDE) a través del Análisis de Varianza de una sola vía, encontrándose diferencias estadísticamente significativas entre las medias de los cuatro grupos, en las cinco palabras estimulo estudiadas. También se aplicó la prueba post hoc de Tukey para identificar los grupos que presentaron las diferencias.

Tabla 6. ANOVA de una sola vía del Indice de Dominancia Evaluativa de la palabra fAMILIA

\begin{tabular}{|c|c|c|c|c|c|c|c|c|}
\hline $\begin{array}{l}\text { Palabra } \\
\text { estímulo }\end{array}$ & Grupos & $n$ & $\bar{x}$ & $D E$ & $\boldsymbol{F}$ & $g l$ & $p$ & $\begin{array}{c}\text { Post hoc } \\
\text { Tukey }\end{array}$ \\
\hline \multicolumn{9}{|c|}{ (Rango de -100 a 100) } \\
\hline Familia & $\begin{array}{l}\text { Usa marihuana (m) } \\
\text { Usa cocaína (c) } \\
\text { Policonsumo }(p) \\
\text { No consumo }(n)\end{array}$ & $\begin{array}{c}44 \\
46 \\
48 \\
148\end{array}$ & $\begin{array}{l}59,7 \\
43,1 \\
39,7 \\
75,5\end{array}$ & $\begin{array}{l}54,8 \\
67,2 \\
50,6 \\
38,0\end{array}$ & 9,5 & 3 &, 0000 & $\mathrm{n}>\mathrm{p}, \mathrm{c}$ \\
\hline
\end{tabular}


En la palabra estímulo familia ( $F=9.5, g l=3, p=.0000)$ las diferencias se presentaron entre los no consumidores de drogas frente los policonsumidores y los usuarios de cocaína (Tabla 6).

Tabla 7. ANOVA de una sola vía del Indice de Dominancia Evaluativa de la palabra AMıgos

\begin{tabular}{|c|c|c|c|c|c|c|c|c|}
\hline $\begin{array}{l}\text { Palabra } \\
\text { estímulo }\end{array}$ & Grupos & $n$ & $\bar{x}$ & $D E$ & $\boldsymbol{F}$ & $g l$ & $p$ & $\begin{array}{c}\text { Post hoc } \\
\text { Tukey }\end{array}$ \\
\hline \multicolumn{9}{|c|}{ (Rango de -100 a 100) } \\
\hline Amigos & $\begin{array}{l}\text { Usa marihuana (m) } \\
\text { Usa cocaína (c) } \\
\text { Policonsumo (p) } \\
\text { No consumo (n) }\end{array}$ & $\begin{array}{c}44 \\
45 \\
48 \\
147\end{array}$ & $\begin{array}{l}36,0 \\
-9,1 \\
10,0 \\
58,9\end{array}$ & $\begin{array}{l}48,8 \\
62,1 \\
55,9 \\
41,2\end{array}$ & 28,1 & 3 & 0000 & $n, m>c, p$ \\
\hline
\end{tabular}

En la palabra estímulo amigos $(F=28.1, g /=3$, $p=.0000)$ se identificaron dos agrupaciones, por un lado los no consumidores y los consumidores de marihuana y por el otro los usuarios de cocaína y los policonsumidores (Tabla 7).

Tabla 8. ANOVA de una sola vía del Indice de Dominancia Evaluativa de la palabra yo

\begin{tabular}{|c|c|c|c|c|c|c|c|c|}
\hline $\begin{array}{l}\text { Palabra } \\
\text { estímulo }\end{array}$ & Grupos & $n$ & $\bar{x}$ & $D E$ & $\boldsymbol{F}$ & $g l$ & $p$ & $\begin{array}{c}\text { Post hoc } \\
\text { Tukey }\end{array}$ \\
\hline \multicolumn{9}{|c|}{ (Rango de -100 a 100) } \\
\hline Yo & $\begin{array}{l}\text { Usa marihuana (m) } \\
\text { Usa cocaína (c) } \\
\text { Policonsumo }(p) \\
\text { No consumo }(n)\end{array}$ & $\begin{array}{c}44 \\
46 \\
48 \\
148\end{array}$ & $\begin{array}{c}37,4 \\
24,3 \\
1,0 \\
64,8\end{array}$ & $\begin{array}{l}48,1 \\
59,8 \\
67,1 \\
33,7\end{array}$ & 25,4 & 3 & ,0000 & $\begin{array}{c}\mathrm{n}>\mathrm{p}, \mathrm{c}, \mathrm{m} \\
\mathrm{m}>\mathrm{p}\end{array}$ \\
\hline
\end{tabular}

En la palabra estímulo yo $(F=25.4, g l=3, p=.0000)$ las diferencias se presentaron entre las medias de los no consumidores de drogas frente a los tres grupos de usuarios, que presentaron un IDE más bajo. Aunque también se presentaron diferencias entre los usuarios de marihuana frente a la de los policonsumidores, dado que estos últimos se ubicaron con un nivel neutro en la evaluación hacia su persona (Tabla 8).

Tabla 9. ANOVA de una sola vía del Indice de Dominancia Evaluativa de la palabra cocaína

\begin{tabular}{|c|c|c|c|c|c|c|c|c|}
\hline $\begin{array}{l}\text { Palabra } \\
\text { estímulo }\end{array}$ & Grupos & $n$ & $\bar{x}$ & $D E$ & $\boldsymbol{F}$ & $g l$ & $p$ & $\begin{array}{c}\text { Post hoc } \\
\text { Tukey }\end{array}$ \\
\hline \multicolumn{9}{|c|}{ (Rango de -100 a 100) } \\
\hline Cocaína & $\begin{array}{l}\text { Usa marihuana (m) } \\
\text { Usa cocaína (c) } \\
\text { Policonsumo (p) } \\
\text { No consumo (n) }\end{array}$ & $\begin{array}{c}32 \\
44 \\
47 \\
133\end{array}$ & $\begin{array}{l}-64,7 \\
-55,7 \\
-46,9 \\
-73,4\end{array}$ & $\begin{array}{l}41,8 \\
43,6 \\
41,1 \\
34,8\end{array}$ & 6,4 & 3 & ,0003 & $n>p$ \\
\hline
\end{tabular}

En la palabra estímulo cocaína $(F=6.4, g /=3$, $p=.0003$ ) las diferencias se presentaron entre los no consumidores de drogas, con un IDE bastante negati- vo frente al de los policonsumidores, el cual también fue negativo, pero de mediana intensidad (Tabla 9). 
Tabla 10. ANOVA de una sola vía del Indice de Dominancia Evaluativa de la palabra MARIHUANA

\begin{tabular}{|c|c|c|c|c|c|c|c|c|}
\hline $\begin{array}{l}\text { Palabra } \\
\text { estímulo }\end{array}$ & Grupos & $n$ & $\bar{x}$ & $D E$ & $F$ & $g l$ & $p$ & $\begin{array}{l}\text { Post hoc } \\
\text { Tukey }\end{array}$ \\
\hline \multicolumn{9}{|c|}{ (Rango de -100 a 100) } \\
\hline Marihuana & $\begin{array}{l}\text { Usa marihuana (m) } \\
\text { Usa cocaína (c) } \\
\text { Policonsumo (p) } \\
\text { No consumo (n) }\end{array}$ & $\begin{array}{c}44 \\
33 \\
45 \\
133\end{array}$ & $\begin{array}{l}5,4 \\
-61,8 \\
-4,7 \\
-67,3\end{array}$ & $\begin{array}{l}53,4 \\
43,0 \\
52,3 \\
35,8\end{array}$ & 46,0 & 3 &, 0000 & $n, c>m, p$ \\
\hline
\end{tabular}

En la palabra estímulo marihuana $(F=46.0, g /=$ $3, p=.0000)$ se identificaron dos agrupaciones, por un lado los no consumidores y los consumidores de cocaína y por el otro los usuarios de marihuana los policonsumidores (Tabla 10).

\section{DISCUSIÓN}

En términos generales los resultados parecen apegarse al postulado de psicopatogénesis de la teoría de la motivación de Maslow (1954/1970). En primer lugar, la confiabilidad de los Indices de Dominancia Evaluativa de la familia, los amigos y el yo (en conjunto) obtiene un nivel aceptable lo cual otorga consistencia a la medición de un constructo.

En segundo lugar, se obtiene validez conceptual, al observarse que los grupos de consumidores presentan menores evaluaciones de la familia, los amigos y el autoconcepto, manifiesto en la presencia de menores promedios en los Indices de Dominancia Evaluativa (IDE), siendo lo contrario en los grupos de usuarios con respecto a las drogas que consumen, en donde mostraron mayores niveles evaluativos.

En este sentido, uno de los hallazgos más relevantes es que, entre los indicadores protectores asociados con la familia, los amigos y el yo se identifica la satisfacción de necesidades deficitatarias (en teoría primero son primero satisfechas por la familia, después participan en los amigos $y$, en conjunto, contribuyen a conformar la autoestima), necesidades que parecieran adecuarse a los cuatro primeros niveles de la pirámide de Maslow (1954/1970a): Fisiológicas, de Seguridad, de Estima y Afectivas, a las cuales se referirá en adelante.

Asimismo, también como protección, se identifica la presencia de sentimientos y actividades placenteras asociados con la satisfacción de estas necesidades, los cuales se relacionan con la activación del circuito de recompensa (Guisa, et al, 2001; Ramos y Fernández, 2000 Volkow et al) al que se referirá en adelante.

Por otra parte, entre los indicadores de riesgo del consumo de drogas, se identifican variables psicosociales adversas, contrarias a la satisfacción de las necesidades identificadas entre los indicadores protectores, por lo que se considera, desde la teoría de la motivación de Maslow, como indicadores de motivación del consumo.

Siendo lo contrario con respecto a los significados psicológicos de las drogas, en donde se encuentran como indicadores motivacionales de riesgo de su consumo, algunas sensaciones que reflejan la activación del circuito de recompensa y la presencia de trastornos emocionales y conflictos familiares.

Como último comentario, sobre las medidas de tendencia central, se observa que la Desviación Estándar de los IDE para todos los grupos resulta muy amplia para los cinco conceptos, siendo ésta o más pequeña para los no consumidores, lo cual muestra lo polarizado que se pueden encontrar los conceptos estudiados para todos los grupos y también que entre los no usuarios la polarización es menor. Lo cual es importante de considerar, dado que resta precisión a la medición de las variables, por lo que se recomendaría una exploración más a fondo de los indicadores identificados, transformándolos en variables escalares.

En la palabra estímulo familia, las diferencias se presentaron entre los grupos de consumidores de cocaína y los policonsumidores frente al grupo de no usuarios de drogas, con una evaluación menor entre los primeros. A la vez que el grupo de usuarios de marihuana no presenta diferencias ni con los otros grupos de consumidores ni con los no usuarios de drogas, lo cual trasluce una menor presencia de factores de riesgo relacionado con una mayor presencia de factores de protección.

Entre los componentes subjetivos positivos de la familia presentes en todos los grupos se encontraron variables psicosociales relacionadas con necesidades de Seguridad: unión, apoyo; la necesidad de Estima: comprensión y la necesidad Afectiva: amor.

Como indicadores psicosociales de protección positivos de la familia se identificaron dos necesidades, una de Seguridad: honestidad y otra Afectiva: amistad, lo cual es afín con la idea de que los lazos fuertes en la familia constituyen factores protectores del consumo de drogas (Munist et al., 1998; Hawkins et al, 1992). 
Se presentaron también como indicadores de protección positivos sentimientos que muestran la activación del circuito de recompensa: alegría y felicidad.

Por otra parte, se identificó un indicador psicosocial protector negativo: el regaño, lo cual es congruente con reportes que vinculan al establecimiento de límites en la familia como factor protector del uso de drogas, así como las normas familiares inconsistentes como factor de riesgo (Hawkins et al., 1992; Pérez y Mejía, 1998), lo que curiosamente coincide con la sabiduría popular que dice: ...si te regañan es porque te quieren.

Otros indicadores psicosociales de protección asociados con la familia fueron las necesidades de Seguridad: trabajo, responsabilidad, convivencia y sinceridad; la necesidad de Estima: educación y la necesidad Afectiva: respeto. Esta última reportada previamente como factor protector del uso de marihuana (García Aurrecoechea y Gracia, 2004).

Entre los indicadores psicosociales de riesgo, asociados con variables familiares y motivación del consumo de drogas se encontraron solamente entre los componentes subjetivos negativos, los cuales resultan incompatibles con los indicadores de protección anteriormente indicados, entre los cuales se destaca peso la presencia de hipocresía, incompatible con la satisfacción de necesidades de honestidad, sinceridad, comprensión y respeto (entre otras), por lo cual, se dificulta la satisfacción de necesidades de deficiencia de Seguridad, Estima y Afectivas.

Por tipo de consumidores sobresale la presencia de agresión en la familia como motivación (o riesgo) del uso de cocaína, lo cual es acorde con los reportes que han identificado como factor de riesgo a la violencia doméstica y al conflicto familiar (Hawkins et al., 1992; Reilly, 1983; Rodríguez-Kuri et al., 2004). Aunque estos datos parecen focalizar esta problemática entre los usuarios de estimulantes, por lo que se impulsa la hipótesis de que el su uso se encuentra precedido de violencia intrafamiliar.

Lo cual presenta congruencia teórica con la identificación como factor de protección la satisfacción de la necesidad de Seguridad: tranquilidad entre usuarios de cocaína reportado en un estudio anterior (García Aurrecoechea y Gracia, 2004), dado que la agresividad es incompatible con la tranquilidad.

Como indicadores psicosociales de motivación del policonsumo de marihuana y cocaína aparece en la familia la falta de atención y las discusiones, lo cual es acorde con los reportes que indican que la falta de cohesión, de relaciones cercanas y de confianza entre padres e hijos como factores de riesgo del consumo de drogas (Hawkins et al., 1992).

Esto es importante, pues es conocido que la falta de atención en la familia puede ser el origen de pro- blemas de salud y de una baja percepción de riesgo entre los hijos, lo cual es consistente con reportes anteriores que indican que la satisfacción de la necesidad Fisiológica: salud y la necesidad Afectiva: cariño conforman factores de protección del policonsumo de drogas (García Aurrecoechea y Gracia, 2004).

Como indicadores psicosociales de motivación del consumo de marihuana asociados con la familia, se encuentra la desintegración y el sentimiento de miedo, que muestran congruencia con la satisfacción de la necesidad de Estima: éxito y de la necesidad Afectiva: respeto como factores protectores (GarcíaAurrecoechea y Gracia), puesto que una familia en estas condiciones no es exitosa y al estar desintegrada no hay condiciones para preservar el respeto.

Lo anterior es congruente con reportes que señalan a la unión padre hijo como factor de protección (Brook, Brook, De la Rosa, Whiteman, Johnson y Montoya, 2000), que focaliza un tipo de integración y de estructura protectora del consumo.

Finalmente, la ausencia de la asociación del consumo de drogas entre las Puntuaciones de Predominio de los Componentes Subjetivos Negativos de la palabra estímulo familia, señalada por la literatura como un factor de riesgo importante (Hawkins et al., 1992; Medina-Mora et al., 2003; Munist et al., 1998; Rodríguez-Kuri, 2004), pudiera ser explicada por las características de las muestras de consumidores, puesto que se conformaron con solicitantes de tratamiento, los cuales muchos (cerca de la mitad), acudieron acompañados por sus familiares, por lo que podría suponerse mayor grado de cohesión y de estructura en las muestras de usuarios estudiados que entre los consumidores que no acuden a tratamiento.

Por el contrario, a la palabra estímulo amigos los tres grupos de consumidores de sustancias la asociaron con el consumo de drogas, lo cual ratifica la identificación del uso de drogas entre el grupo de pares y amigos como factor de riesgo del consumo (MedinaMora et al., 2003; Munist et al., 1998; Rodríguez-Kuri et al., 1999).

Llama la atención que los usuarios de marihuana y los no consumidores de drogas no se diferencian en el IDE de los amigos, pero ambos se diferencian de los consumidores de cocaína y policonsumidores, quienes presentan IDE alrededor de lo neutro, en lo que parece una mayor presencia de indicadores de riesgo, asociado a una mayor presencia de indicadores de protección.

Entre los componentes subjetivos positivos de los amigos presentes en todos los grupos, se encontraron variables psicosociales relacionadas con necesidades de Seguridad: sinceridad, ayuda y apoyo y; una Afectiva: la propia amistad. 
También todos los grupos manifestaron indicadores que reflejan sentimientos y acciones sociales relacionadas con la activación del circuito de recompensa: alegría, juego y diversión (recuérdese que en México los juegos de azar con apuesta están prohibidos).

Como indicadores de protección se encontraron necesidades psicosociales de Seguridad: unión, confianza y convivencia; de Estima: comprensión y estudio; Afectivas: amabilidad, respeto y cariño.

Entre los indicadores psicosociales de riesgo, asociados con los amigos y motivación del consumo de drogas, se encontraron algunos componentes subjetivos positivos. Entre ellos, aparecen las platicas, el cotorreo, la fiesta y el relajo, siendo actividades que pueden relacionarse con la activación del circuito de recompensa, aunque también con el consumo de drogas, parecidos a la diversión, juego y alegría indicados anteriormente.

Un indicador particular de riesgo entre los grupos de monousuarios (cocaína o marihuana) es considerar a los amigos como hermandad, satisfaciendo necesidades que los lazos familiares no son capaces de lograr, por ser agresivas (hipócritas o violentas) o desintegradas (pasivas).

Otros indicadores positivos que representan riesgo para el uso de marihuana son salidas, paseos y viajes, actividades acordes con lo reportado por Gracia et al. (2002) como un factor de riesgo individual para los usuarios de esta sustancia la necesidad Fisiológica: búsqueda de sensaciones. Como indicadores de riesgo de tipo negativo se encuentra considerar que los amigos "no existen", lo cual muestra crudamente un esquema mental que disminuye la capacidad satisfactora de este medio microsocial.

Como indicadores de riesgo del uso de cocaína y del policonsumo se encuentra considerarlos hipócritas o convenencieros, lo cual refiere un tipo de violencia encubierta al interior de estos grupos microsociales, afín con la referida para el núcleo familiar de los usuarios de cocaína y acorde también con la pertenencia a redes sociales disfuncionales (Rodríguez-Kuri et al., 1999), relacionado con lo caro que resulta mantener el consumo del alcaloide.

Entre indicadores de riesgo negativos del uso de marihuana se encuentra considerar a los amigos traidores, lo cual pudiera también reflejar lo frágil que puede ser una expectativa de hermandad, proveniente de deficiencias de satisfacción de una estructura familiar desintegrada.

Con respecto a la palabra yo, los no consumidores presentaron un concepto de sí mismos notoriamente mayor que los tres grupos de consumidores, con un IDE de 65. Entre los grupos de usuarios, es notable como los policonsumidores presentan un IDE muy bajo, cercano a cero, el cual se alcanza a diferenciar del IDE de 37 de los usuarios de marihuana.

Entre los componentes subjetivos positivos relacionados con el autoconcepto, en todos los grupos se encontraron variables psicosociales relacionadas con necesidades de Seguridad: bondad y trabajar; la necesidad de Estima: estudiar y; la necesidad Afectiva: amistad.

También todos los grupos destacaron la alegría entre sus componentes subjetivos positivos, mostrando sentimientos de satisfacción consigo mismos y la manifestación de cierta activación del circuito de recompensa asociado con su persona.

Como indicadores positivos de protección del consumo de drogas, para los tres tipos de consumo de drogas, aparece la necesidad de Seguridad: libertad y la necesidad Afectiva: respeto, presente también como indicador de protección tanto en la familia, como con los amigos, aparece ahora interiorizada como indicador individual de protección.

Otros indicadores de protección positivos son las necesidades de Seguridad: tranquilidad, sinceridad, responsabilidad y ayudar; la necesidad de Estima: inteligencia y; las necesidades Afectivas: amory amabilidad. Asimismo, como indicadores de protección positivos se encuentran las actividades lúdicas de cantar, bailar y jugar, relacionadas con la activación del circuito de recompensa.

Por otra parte, como indicadores de riesgo de motivación del consumo, sobresalen los de tipo negativo, entre los que se encuentra el propio consumo de drogas, inclusive definirse como drogadictos. Otros indicadores de riesgo negativos son: a) alejarse y la soledad; b) sentimientos de tristeza, inseguridad, miedo y depresión; c) estados mentales de confusión; d) rebeldía, insatisfacción; e) agresividad, antisocial, problemas.

A diferencia de los otros grupos, el poliusuario se caracterizó por asociarse con una plétora de conceptos auto denigratorios, (tarado, idiota, cochino, rata, flojo, no valgo, no merezco) los cuales pueden asociarse con estados depresivos (de acuerdo con planteamientos cognoscitivos) y que pudieran haber sido interiorizados a partir de la falta de atención en la familia.

Por su parte, el monousuario de cocaína parece concebirse particularmente agresivo, lo que pudiera focalizar en este grupo de usuarios de estimulantes los problemas de conducta; adicionalmente, como un indicador de riesgo positivo, se consideran fuertes, lo que podría ser una condición necesaria para ser agresivos, aunque también se asocian con insatisfacción y con miedo. 
A diferencia, el monousuario de marihuana se significa como rebelde, lo cual aparece factible en el contexto de una familia desintegrada.

Con respecto los IDE de la palabra estímulo cocaína, destaca que para todos los grupos estos fueron negativos, lo que asegura una percepción negativa generalizada de la sustancia, pero ésta percepción resulta menos negativa entre los policonsumidores que entre los no usuarios, lo que permite calificarlos como más temerarios en su mundo subjetivo.

Como indicadores protectores de su consumo aparecen solamente los de tipo negativo, considerando a la sustancia como una enfermedad, perjudicial, peligrosa, que no les gusta, que es una droga mala, cara, produce adicción y conduce a la soledad, lo cual confirma una mayor percepción de riesgo del consumo del alcaloide entre los no usuarios de drogas.

A diferencia como indicadores motivacionales (de riesgo) del consumo de cocaína presentes en los mono y policonsumidores de esta sustancia se encontraron tanto de tipo positivo como negativo. Entre los primeros se encuentra la satisfacción de la necesidad de Seguridad: tranquilidad, así como sensaciones que permiten identificar la activación del circuito de gratificación: les gusta, es rica, y da placer.

Con respecto a la palabra estímulo marihuana, la presencia de IDE negativos entre los no consumidores y consumidores de cocaína frente a los IDE neutros de los usuarios de marihuana y los policonsumidores, indica la presencia de mayores proporciones de asociaciones positivas en el mundo subjetivo de los mono y policonsumidores de marihuana hacia esta palabra estímulo.

Como componentes subjetivos protectores de su consumo, se encontraron de tipo negativo, como considerarla nociva, un mal hábito, un vicio, una enfermedad y lo asociaron con un sentimiento de desesperación, lo que hace ver una mayor percepción de riesgo entre los no consumidores de esta sustancia.

Entre los indicadores individuales negativos de riesgo asociados con la motivación de consumo de cocaína, se encontraron algunos trastornos emocionales: ansiedad, tristeza, depresión, miedo, nervios, desesperación, pánico y paranoia, así como la asociación con transgresiones a la ley que implican el riesgo de ir a la cárcel.

Entre los indicadores motivacionales de riesgo del consumo de marihuana presentes en los usuarios de marihuana y policonsumidores, se encontrararon de tipo positivo y negativo. Entre los primeros se ubicó la necesidad Fisiológica: búsqueda de sensaciones, así como la asociación con sensaciones que reflejan la activación del circuito de gratificación: ayuda a la diversión, produce placer, es agradable, te ayuda al cotorreo, produce risa y alegría.
Finalmente, entre los indicadores motivacionales negativos de riesgo del consumo de marihuana, se encontraron los conflictos y los problemas, sobre todo de tipo familiar, lo que es acorde con autores que asocian el consumo de drogas con problemáticas familiares (Hawkins et al., 1992; Rodríguez-Kuri et al., 2004).

\section{REFERENCIAS}

Díaz-Guerrero, R. y Szalay, L. B. (1993). El mundo subjetivo de mexicanos y norteamericanos. México: Ed. Trillas.

Díaz-Guerrero, R. y Díaz-Loving, R. (2001). El origen psicológico de los valores. Revista de Psicología Social y Personalidad, 17, 91-101.

García-Aurrecoechea, V. R. y Gracia, S. E. (2004). Grado de satisfacción de necesidades y consumo actual de mariguana y/o cocaína en jóvenes varones solicitantes de tratamiento. México: Centros de Integración Juvenil, Dirección de Investigación y Enseñanza, Subdirección de Investigación. Informe de Investigación 04-10.

González-Sánchez, J. D., García-Aurrecoechea, V. R. y Córdova, A. (2004). Uso de sustancias entre adolescentes y su relación con síntomas de depresión y su percepción de sus relaciones familiares. Informe de Investigación 04-03 México: Centros de Integración Juvenil, A. C.

Gracia Gutiérrez de Velazco, S. E., Saldívar-Garduño, A., Y Contreras-Ibañez, C. C. (2002). Validación de la escala de búsqueda de sensaciones: Rasgo de personalidad y su importancia en la adicción a las drogas. La Psicología Social en México, Vol. 9, 411-418.

Guisa, V. M., Díaz-Barriga, L, Sánchez-Huesca, R. y Sousa, M. (2001). Farmacoterapia de los síndromes de intoxicación y abstinencia por psicotrópicos. México: Centros de Integración Juvenil, A. C.

Hawkins, J. D., Catalano, R. F. y Miller, J. Y. (1992). Risk and protective factors for alcohol and other drug problems in adolescence and early adulthood: Implications for substance abuse prevention. Psychological Bulletin, 112, 64-105.

Llopis, LI. (2001). Dependencia, intoxicación aguda y síndrome de abstinencia por cocaína. En F. Pascual, M. Torres y A. Calafat (Eds.), Monografía cocaína (pp. ). Adicciones, 13, suplemento 2, 147-165.

Maslow, A. (1954/1970a) A theory of human motivation. En A. Maslow (Ed.), Motivation and personality (pp. 35-57). New York and London: Harper and Row Publishers.

Maslow, A. (1954/1970b) Psychopathogenesis and the theory of threat. En A. Maslow, Motivation and personality (pp. 105-115). New York and London: Harper and Row Publishers.

Medina-Mora, M. E., Cravioto, P., Villatoro, J., Fleiz, C., Galván-Castillo, F. y Tapia-Conyer, R. (2003). 
Consumo de drogas entre adolescentes: resultados de la Encuesta Nacional de Adicciones, 1998. Salud pública Méx. [online]. Vol.45. suppl.1: 16-25. En: http://www.scielosp.org/scielo.php?script=sci_ arttext\&pid=S0036-36342003000700005\&lng=en\& $\mathrm{nrm}=\mathrm{iso}$

Munist, m., Santos, H., Kotliarenco, A., Suárez, N., Infante, F. y Grotberg, (1998). Manual de identificación y promoción de la resiliencia en niños adolescentes. Organización Panamericana de la Salud, Organización Mundial de la Salud, Fundación W. K. Kellogg.

Pérez, A. y Mejía, I. (1998). Patrones de interacción de familias en las que no hay consumidores de sustancias psicoactivas. Adicciones 10, 111-119.

Ramos Atance, J. A., Fernández Ruiz, J. J. (2000). Sistema cannabinoide endógeno: ligandos y receptores acoplados a mecanismos de transducción de señales. En J. Bobes y A. Calafat (Eds.), Monografía Cannabis. Cannabis sativa. Adicciones, Vol. 12, suplemento 2, 59-82. (www.irefrea.org/Publications/Spanish/ cannabis.pdf).
Reilly, D. M. (1983). Family factors in the etiology and treatment of youthful drug abuse. En: T. J. Glynn (Ed.). Drugs and the family. NIDA Research Issues Num. 29, 125-127.

Rodríguez-Kuri, S. E. (2004). Factores familiares y de pares asociados al consumo de drogas. Tesis de maestría no publicada. México: Universidad Nacional Autónoma de México.

Rodríguez-Kuri, S., Arellanes, J. L., Díaz-Negrete, D. B. y González-Sánchez, J. D. (1999). Ajuste psicosocial y consumo de drogas. CIJ, Informe de Investigación 9727. México: Centros de Integración Juvenil, A. C.

Szalay, L. B. y Bryson, A. (1974) Psychological Meaning; Comparative Analysis and Theoretical implications. Journal of Personality and Social Psychology, 30, 860870.

Volkow, M.D., Fowler, J. S. y Wang, G. J. (2003). The addicted human brain: insights from imaging studies. J. Clin Invest 111 (10) 1444-1451. 